\title{
Impact des termites dans les champs paysans de riz et de mais en savane sub-soudanienne (Booro-Borotou, Côte-d'Ivoire)
}

\author{
Alexandre A. AkPeSse ${ }^{1 *}$, Philippe K. Kouassi ${ }^{1}$, Yao TANO ${ }^{1}$. \& Michel Lepage ${ }^{2}$ \\ ${ }^{1}$ UFR Biosciences, Université de Cocody, 22 BP 582 Abidjan 22- Côte d'Ivoire \\ 2 IRD-Ouagadougou 01 BP 182 Ouagadougou-Burkina Faso \\ *Auteur pour les correspondances (Email : alexakpesse@ hotmail.com / alexakpesse@ yahoo.fr) \\ Reçu le 16-08-2006, accepté le 29-02-2008.
}

\begin{abstract}
Résumé
Les termites appartiennent au groupe des arthropodes et jouent un rôle prépondérant dans les régions tropicales et sub-tropicales. L'objectif de la présente étude est d'évaluer les dégâts causés par les termites dans les cultures paysannes de riz (Oryza sativa L.) et de maïs (Zea mays L.). Pour se faire, un échantillonnage préliminaire a été entrepris dans le milieu naturel (savane arbustive) en vue d'inventorier les espèces de termites présentes dans le milieu afin de les utiliser comme témoin durant cette étude. Six espèces de termites ont été recensées dans le milieu naturel contre respectivement 7 et 4 dans les champs de riz et de maïs. Les ouvriers de termites champignonnistes (Microtermes $s p$, Ancistrotermes sp) et les xylophages (Amitermes sp.) ont été fréquemment rencontrés dans le système racinaire et à l'intérieur des tiges des deux cultures et sont considérés comme responsables des dégâts. Certains termites souterrains (Microtermes sp, Ancistrotermes sp et Amitermes sp) endommagent les racines et les tiges de maïs. Ils sont par conséquent responsables de la verse des plantes après sénescence. D'autres termites comme Pseudacanthotermes sp et Macrotermes $s p$ construisent des placages sur les plants qu'ils utilisent pour les attaquer. Aucun dégât n'a été attribué aux termites humivores Cubitermes $s p$.
\end{abstract}

Mots clés : Termites, dégâts, champs paysans, riz, maïs.

\begin{abstract}
Impact of termites on peasant rice and maize in sub-soudanese savannah (Booro-Borotou, Côte-d'lvoire)
\end{abstract}

Termites belong to the athropod group and play a tremendous role in tropical and sub-tropical regions. The aim of this study was to assess the damages caused by termites to the rice (Oryza sativa $L$ ) and corn (Zea mays $L$.) in farmer fields. To reach this goal, a preliminary sampling was undertaken from the natural environment (shrub savannah) in a view of inventorying termites species to be used in this investigation. Six species of termites were identified in the shrub savannah and 7 and 4 while were collected in the rice and corn farms, respectively. The fungus-growing worker termites (Microtermes sp, Ancistrotermes $\mathrm{sp}$ ) and wood-feeders ones (Amitermes $\mathrm{sp}$ ) were frequently met in the root system and in the stems of the two crops and they were identified as those responsible for damages. Some subterranean termites (Microtermes sp. Ancistrotermes sp and Amitermes sp) damage the roots and stems of corn. They are subsequently responsible for the fall of crops after senescence. Other termites such as Pseudacanthotermes $\mathrm{sp}$ and Macrotermes sp build up outside layers on crop that they use to infest it. No soil-feeding termite (Cubitermes $\mathrm{sp})$ was held responsible for any damage.

Keys -words: Termites, damage, peasant, rice,corn. 


\section{Introduction}

Les termites (Isoptères) comptent environ 2600 espèces et 281 genres (Kambhampati \& Eggleton, 2000). Ils jouent un rôle prépondérant dans l'évolution des sols tropicaux (Carter \& Murwira, 1995 ; Lavelle et al., 1997 ; Bignell \& Eggleton, 2000). Environ $10 \%$ de ces espèces de termites ont des impacts négatifs dans les habitations et sur les cultures (Fowler \& Forti, 1990 ; Mill, 1991 ; Constantino, 2002). Les pertes de récolte engendrées par ces termites sont énormes de l'ordre de 20 à $45 \%$ (Logan et al., 1990 ; Wood \& Pearce, 1991).

En Afrique de l'ouest, en général, et en Côted'Ivoire, en particulier, où le riz et le maïs constituent les aliments de base des populations (Séré, 1990), les dégâts des termites sur ces cultures sont avérés (Sands, 1973 ; Cissé, 1991 ; Akpesse, 2001). Face à cette situation, les agriculteurs modernes ont recours à l'usage d'engrais et de substances insecticides pour améliorer la fertilité des sols et optimiser la production de ces cultures. Ce n'est pas le cas des paysans, qui dans l'incapacité de débourser des fortes sommes pour acheter des engrais et pesticides doivent trouver des méthodes de luttes alternatives (Iroko, 1996).

De nombreuses études ont été réalisées sur les termites en plantations expérimentales et industrielles (Cissé ; 1991, Han et al, 1998 ; Akpesse, 2001). Cependant, très peu de travaux traitent des dégâts des termites en plantations paysannes de Côte d'Ivoire. Cette étude fait suite à des travaux réalisés en parcelles expérimentales ayant subi des traitements chimiques à base de fipronil et chlorpyrifos-éthyl (Akpesse et al., 2001 et 2004). Une nouvelle approche serait de mettre en place des méthodes de lutte alternatives contre les termites ravageurs.

L'objectif de cette étude est d'évaluer les dégâts causés par les termites dans les champs de riz et de maïs. Sa réalisation a nécessité dans un premier temps à faire l'objectif principal est subdivisé en plusieurs objectifs spécifiques: l'inventaire et l'estimation de la diversité spécifique et fonctionnelle des termites dans les champs de riz et de maïs ensuite une description des modalités d'attaques des termites ravageurs et enfin une estimation des dégâts sur les cultures.

\section{Matériel et Méthodes}

\subsection{Site d'étude}

Le site de Booro-Borotou ( $8^{\circ} 28^{\prime} 45^{\prime \prime} \mathrm{N} ; 7^{\circ} 34^{\prime} 42 \mathrm{~W}$ ) est localisé en zone de savane préforestière humide du secteur sub-soudanais. Le climat est de type subtropical à deux saisons. Les précipitations enregistrées à la station météorologique de Touba de 1930 à 1981, ont montré que la moyenne des pluies est de 1334,1 $\mathrm{mm}$ (Pham, 1990). Les données pluviométriques des années 1998, 1999 et 2000 de la région de Touba révèlent une moyenne annuelle de 1278,5 $\mathrm{mm}$, ce qui montre une région relativement bien arrosée. Les températures moyennes mensuelles oscillent entre $25,3^{\circ} \mathrm{C}$ et $31,3^{\circ} \mathrm{C}$ (Projet soja). Les sols correspondent à deux grands domaines: le domaine ferralitique et le domaine ferrugineux hydromorphe (Fritsch \& Planchon, 1987). La parcelle expérimentale est localisée en milieu paysan sur des sols ferralitiques de plateau et de flanc de cuirasse (unité S-1 selon Fritsch \& Planchon, 1987). Les sols présentent une teneur en matière organique de $3,35 \%$, des taux de $2,63 \%$ pour le carbone, $1,85 \%$ pour l'azote et de $0,04 \%$ pour le phosphore (Boga et al., 2000).

\subsection{Matériel végétal}

Deux cultures ont été testées sur le site : le riz Oryza sativa L. (Poaceae) et le maïs Zea mays L. (Poaceae). Les variétés de riz à cycle court (var. IAC 170, originaire du Brésil) et de maïs (var. Ferké II) également à cycle court (100 à 120 jours) sont toutes deux couramment cultivées par les paysans de cette zone. Les semis ont eu lieu dans le mois de juin 2001.

\subsection{Méthodes}

\subsubsection{Modalités d'attaques des termites dans les cultures}

Pour caractériser les attaques des termites dans les champs, une méthode destructive des plants a été utilisée une semaine après que les paysans aient réalisés leur récolte. La méthode destructive a consisté à déterrer à l'aide de dabas des pieds de maïs et des touffes de riz choisis de façon aléatoire dans chaque champ (Wood et al., 1977 ; 
Sarr, 1999 ; Sekamatte et al., 2001 ). Les racines, les tiges et les feuilles de chaque pied ou touffes ont été séparées. La présence ou l'absence de termites à été notées au niveau de chaque partie du végétal. L'attaque des cultures par les termites a été évaluée à partir d'une échelle de notation basée sur l'étendue des galeries observées tant au niveau des racines que des tiges des deux plantes en vue de voir l'ampleur des dégâts.

\subsubsection{Impact des termites dans les champs}

Cette étude a été réalisée dans 10 champs de riz et 20 champs de maïs d'une superficie d'environ 1 ha chacun. Les champs ont été choisis au hasard en tenant compte de la toposéquence en pente. Les termites ont été échantillonnés à l'aide d'unité de surface (Sands, 1973 ; Brenière, 1983). Pour le riz dont la plante pousse sous forme de thalles, les touffes formées étant au niveau du sol, l'échantillonnage sur une surface donnée est plus appropriée (Fall, 2000). Les plants sur une surface de $1000 \mathrm{~m}^{2}(10 \times 100 \mathrm{~m})$ ont été arrachés à l'aide de dabas et une fouille systématique des différentes parties du végétal (racines, tiges) et du sol a été réalisée.

Pour ce qui concerne le maïs, l'échantillonnage a été réalisé sur un nombre total de 2000 pieds. Cet échantillonnage est plus approprié compte tenu du fait que les semis du maïs se font par poquet (Wood et al., 1977 ; Sarr et al., 2001 ; Sekamatte et al., 2001). En se référant à l'échantillonnage du riz qui se fait par unité de surface, dans les champs de maïs, 2000 pieds à raison de 100 par champ ont été déterrés et fouillés au niveau des racines et des tiges.

\subsubsection{Echantillonnage des termites en milieu naturel (savane)}

Au préalable, une parcelle témoin, située dans la savane arbustive a été échantillonnée selon la méthode des transects afin d'avoir une estimation de la richesse spécifique des populations de termites dans le milieu naturel (Lepage, 1974 ; Eggleton et al., 1995 ; Fall et al. 2000). Un itinéraire de longueur connue (1 km) et découpé par tranches successives de $100 \mathrm{~m}$ a été parcouru en ligne droite. Une fouille générale le long de ce transect a eu lieu. Cette méthode est également pratiquée pour l'échantillonnage des fourmis et permet de répertorier tous les nids présentant des signes extérieurs et les points de contact des termites avec le layon sur la litière (Lévieux, 1963 ; Diomandé, 1983).

\subsubsection{Traitements statistiques}

Les tests non paramétriques de Kruskal Wallis ont permis de rechercher des différences entre les taux d'abondance des différents genres de termites au sein des champs de riz et de maïs. Par la suite, nous avons utilisé, le test de comparaison de Bonferroni (Sokal et al., 1995) pour la comparaison des différentes moyennes.

Le test de Mann-Whitney a été utilisé pour étudier la pression parasitaire des termites au niveau des champs de riz et de maïs. Les attaques de la racine et de la tige étant indissociables chez le riz nous avons utilisé le test de parité (Pairedtest) pour comparer la pression parasitaire au niveau de ces deux parties du végétal (Rice, 1989 ; Carmélia et al., 2003).

Pour analyser la diversité des populations de termites, nous avons calculé les indices de diversité (Indice de Shannon) et d'équitabilité. Une analyse de variance (ANOVA) a permis de comparer les indices de diversité moyens, suivi d'un test de Tukey pour la séparation de ces différents indices.

Par la suite, nous avons calculé l'indice de similarité selon la formule de Jaccard (Legendre \& Anderson 1999 ; Legendre \& Gallagher, 2001). Le pourcentage de similarité (PS) traduit la somme des plus faibles proportions de chaque espèce de deux communautés alors que le coefficient de Jaccard est un indice qui met l'accent sur les différences entre les biotopes.

PS $=\sum$ [faible proportion de chaque espèce des deux sites] $x 100$

$$
S_{T A}(i, j)=\frac{a}{a+b+c}
$$

\section{PS : Pourcentage de Similarité}

a : nombre d'espèces communes au relevé i et au relevé $j$

$b$ : nombre d'espèces présentes seulement dans le relevé $i$

$c$ : nombre d'espèces présentes seulement dans le relevé $j$ 


\section{Résultats}

\subsection{Diversité des termites dans les plantations paysannes}

Dans les champs de riz, 7 espèces de termites appartenant aux principaux groupes trophiques (groupes fonctionnels) de termites ont été recensées (Tableau 1) : Les champignonnistes (Microtermes sp, Ancistrotermes sp, Pseudacanthotermes sp et Macrotermes $s p$ ) ; les humivores (Cubitermes $s p$ ) ; les xylophages (Amitermes $s p$ ) et les fourrageurs (Trinervitermes $s p)$. Parmi ces groupes de termites, les champignonnistes et les xylophages sont les plus fréquents (Tableau 1 ).

Dans les champs de maïs, le nombre d'espèces (4) est inférieur à celui du riz. Microtermes $s p$, Ancistrotermes sp, Macrotermes $s p$ et Pseudacanthotermes sp appartiennent au groupe des champignonnistes alors que les humivores sont représentés par Cubitermes sp (Tableau 1). Ancistrotermes et Microtermes sont souvent rencontrés au niveau des racines des deux cultures avec des proportions respectives de 17,9\% $\pm 3,47$ et $18,9 \% \pm 3,0$ en culture de riz contre $25 \% \pm 4,03$ et $19,4 \% \pm 1,99$ en culture de maïs (Tableau 1 ). Les proportions de ces deux genres précités
(Ancistrotermes et Microtermes) sont significativement supérieures à celles des genres Cubitermes, Trinervitermes, Pseudacanthotermes et Macrotermes dans le champ de riz (Fig 1; Kruskal-Wallis test, $H=32,2 ; d f=6, P<0,0001$ ). Les proportions de termites présents sont également significativement supérieurs à celles des genres Cubitermes, et Pseudacanthotermes dans le champ de maïs (Fig 2 ; Kruskal-Wallis test, $H=55,2, d f=3, P<0,0001)$.

Dans le milieu naturel (savane arbustive), 6 espèces de termites issus des 4 groupes trophiques similaires à ceux du riz ont été recensées (Tableau 1). Au niveau spécifique, Aderitotermes sp (Humivore) a été observé en savane arbustive, contrairement à Cubitermes $s p$ dans les champs.

Pour ce qui concerne la diversité moyenne, les peuplements de termites des champs de riz $(H$ $=1,62 ; E=0,62$ ) sont plus diversifiés que ceux du milieu naturel $(H=0,67 ; E=1,49)$ et des champs de maïs $(H=0,86 ; E=1,16)$. II existe une différence hautement significative entre les diversités moyennes de ces trois biotopes $(\mathrm{P}<$ 0,05) (Tableau 2).

La similarité calculée entre les deux cultures est de $69 \%$ pour un coefficient de Jaccard de $26,7 \%$.

Tableau 1 : Proportions moyennes des termites (en \%) au niveau des racines et des tiges des champs de riz et maïs à Booro- Borotou

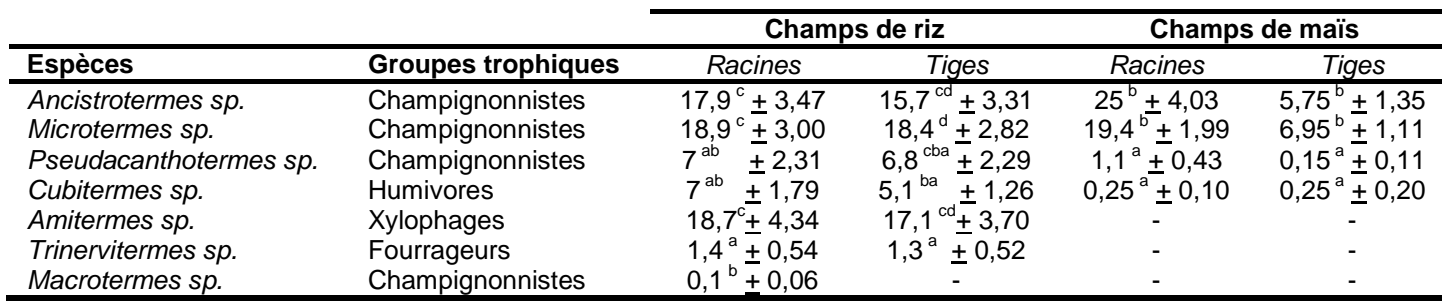

Les moyennes suivies d'une même lettre ne diffèrent pas significativement au seuil de $5 \%$ selon le test de Bonferroni.

Tableau 2 : Indice de diversité moyen de Shannon et d'équitabilité moyen des champs de et riz et maïs de BooroBorotou

\begin{tabular}{lccc} 
& Milieu naturel & Champs de riz & Champs de maïs \\
\cline { 2 - 4 } Richesse spécifique & 6 & 7 & 4 \\
Indice de diversité de Shannon & $0,67^{\mathrm{a}}$ & $1,62^{\mathrm{b}}$ & $0,86^{\mathrm{a}}$ \\
Equitabilité & $1,49^{\mathrm{a}}$ & $0,62^{\mathrm{b}}$ & $1^{\mathrm{a}}, 16^{\mathrm{a}}$ \\
\hline
\end{tabular}

Les moyennes suivies d'une même lettre ne diffèrent pas significativement au seuil de 5\% selon le test de Tukey. 


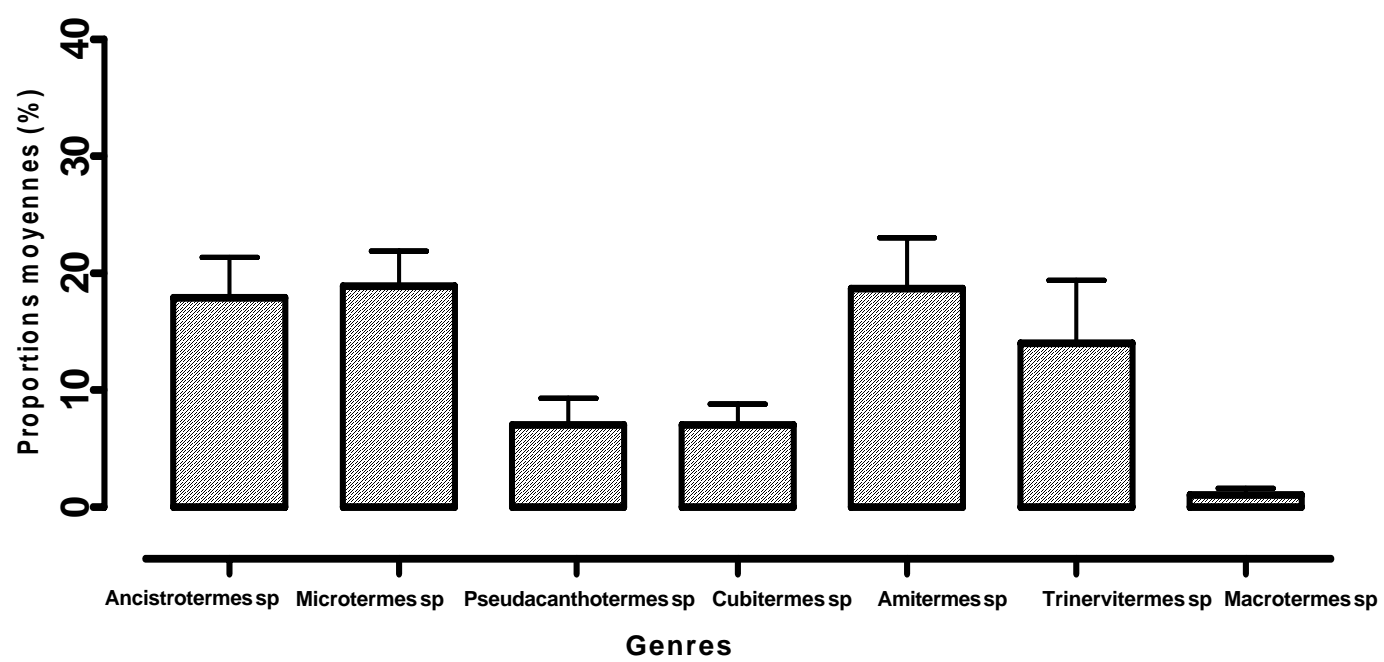

Figure 1 : Présence moyenne des termites au niveau des racines de riz (Kruskal-Wallis test, $\mathrm{H}=32,2 ; d f=6, P<0,0001$ )

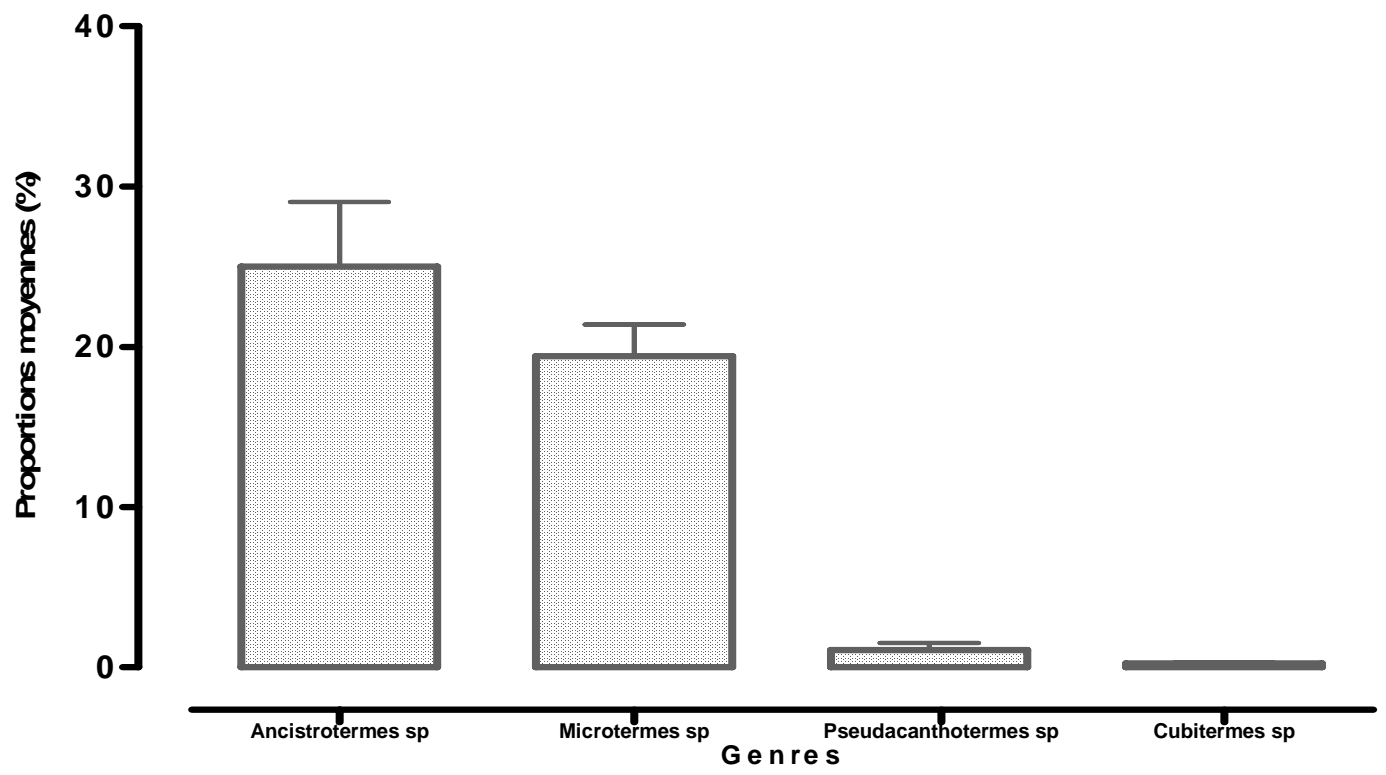

Figure 2 : Présence moyenne des termites au niveau des racines de maïs

(Kruskal-Wallis test, $H=55,2, d f=3, P<0,0001$ ) 


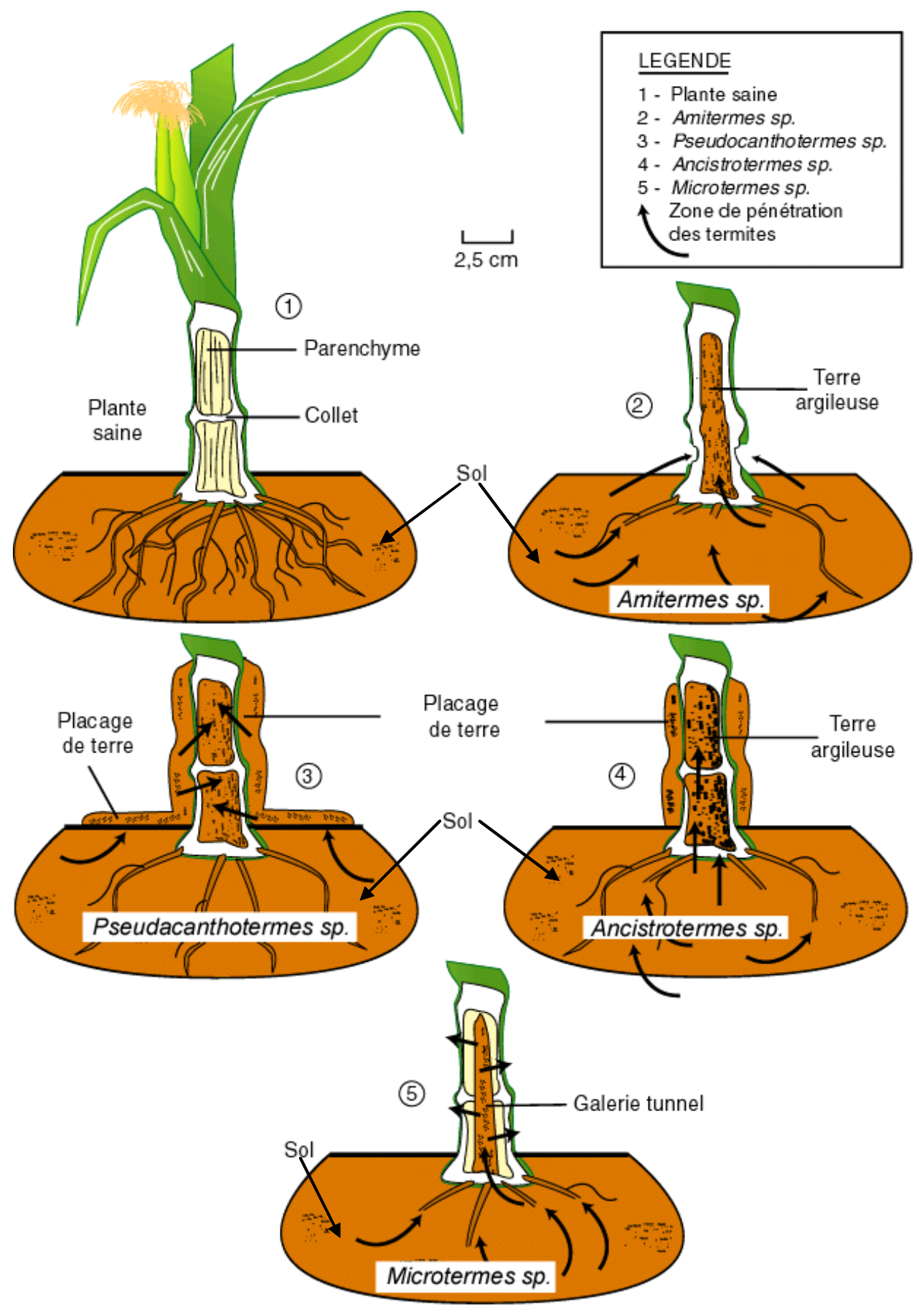

Figure 3 : Modalités d'attaque des plants de maïs par les espèces de termites 


\subsection{Modalités d'attaques des termites aux plants de cultures}

Les modalités des attaques sont différentes selon les espèces (Fig. 3).

- les termites du genre Amitermes sp (Fig. 3.2) coupent les racines à la base et détruisent le parenchyme de la tige ; l'intérieur du collet est remplacé par la terre argileuse. Les attaques ont également lieu à l'extérieur de la tige lorsque le termite consomme le cortex externe pour pénétrer à l'intérieur ;

- les termites Pseudacanthotermes sp (Fig. 3.3) construisent plutôt des placages de terre sur le sol et sur la plante. Ces placages leur servent de protection pour attaquer la plante. Le parenchyme de la tige est détruit et l'intérieur du collet est remplacé par la terre argileuse. Les attaques se déroulent de l'extérieur vers l'intérieur de la tige ;

- les termites Ancistrotermes sp (Fig. 3.4) attaquent généralement les racines en premier ; celles-ci sont coupées à la base et les termites pénètrent à l'intérieur de la tige. Au niveau de cette tige, ces termites détruisent le parenchyme cortical en consommant le cambium interne. L'intérieur du collet est alors remplacé par la terre argileuse, en quantité relativement importante. Pour les espèces de ce genre, un autre type d'attaque est fréquent. Elle se manifeste par la construction de placages externes sur la tige, lesquels servent de passage pour attaquer le végétal de l'extérieur vers l'intérieur, en consommant successivement le cortex externe de la tige, le parenchyme interne et le cambium de la tige. Le collet totalement détruit est remplacé comme dans le premier mode d'attaque par de la terre ;

- les termites Microtermes sp (Fig. 3.5) sont de petites tailles et très mobiles. Ils coupent les racines à la base et progressent ensuite vers l'intérieur de la tige au niveau de laquelle ils construisent une galerie interne qui leur sert de passage. Une fois à l'intérieur de la tige, les termites consomment le cambium et le parenchyme interne de la tige, de l'intérieur vers l'extérieur. Le collet évidé est progressivement comblé avec de la terre.

\subsection{Impact des termites dans les plantations paysannes}

\subsubsection{Champs de riz}

La proportion des pieds attaqués par les termites sur l'ensemble des champs (surface totale de 10 ha) est de $61,9 \% \pm 3,67$ au niveau des racines contre $20,4 \% \pm 3,47$ au niveau des tiges. Une comparaison des attaques des termites entre ces deux parties du végétal par le test de parité (Paired-t test) montre une différence très hautement significative au niveau des racines au seuil de $5 \%\left(t^{\prime}=17,85\right.$ et $\left.P<0,0001\right)$. Par contre, la pression parasitaire exercée par les termites est aussi importante sur le riz que sur le maïs $\left(K h i^{2}=0,9 ; d d l=1\right.$ et $\left.P=0,766\right)$. Bien que présents dans les champs, les termites humivores (Cubitermes $s p$ ) n'occasionnent pas de dégâts.

\subsubsection{Champs de maïs}

Dans les champs de maïs prospectés (surface total de $20 \mathrm{ha}$ ), les attaques des termites s'élèvent à $60,8 \pm 4,08 \%$ au niveau des racines contre $40 \pm 5,99 \%$ au niveau des racines. La différence des attaques des tiges est hautement significative comparativement à celle des racines par le test de Mann-Whitney $\left(U^{\prime}=312\right.$ et $P=$ 0,0026 ). Les attaques des racines et des tiges de maïs sont faiblement corrélées à la nature des champs; $a ́=0,01$; ddl $=19$ et $P<0,01$ ).

\section{Discussion}

Nos résultats ont révélés 7 et 4 espèces de termites respectivement dans les champs de riz et de maïs contre 6 espèces dans le milieu naturel. Les espèces rencontrées dans le milieu naturel, colonisent généralement les champs de riz. Seuls Macrotermes sp et Cubitermes sp n'ont pas été observées dans la savane arbustive lors de notre échantillonnage car ces termites ont des nids épigés. La présence de ces espèces s'expliquerait par la forte densité des termitières de Macrotermes et de Cubitermes dans les 
zones au voisinage des champs paysans (Tano, 1993 ; Kouassi, 1999). La baisse du nombre d'espèces de termites dans les champs s'expliquerait par les traitements des sols avant la mise en cultures. En effet, les paysans défrichent et brûlent la végétation avant les semis, ce qui favoriserait la disparition des groupes des termites humivores et fourrageurs, suivie de l'installation et du développement des colonies des espèces à habitat souterrain (Macrotermitinae) et des termites xylophages (Amitermitinae) (Wood et al., 1977 et 1980 ; Kooyman \& Onck, 1987 ; Umeh et al., 2001). Aussi, seuls subsistent dans les champs de maïs, Microtermes et Ancistrotermes du fait de leurs nids souterrains ubiquistes et de leur capacité de migrer dans les profondeurs du sol lors de la sécheresse (Lepage, 1974 ; Wood, 1996). II faut ajouter que l'espèce végétale utilisée comme plante de couverture (arachide, tabac et neem) pourrait jouer un rôle important dans la répulsion ou l'attirance des termites (Raymundo, 1986 ; Wardell, 1987 ; Cissé, 1991). Les termites en général, et les champignonnistes en particulier, constituent l'essentiel de la macrofaune du sol en milieu de culture de riz et de maïs sur ce site (Akpesse et al., 2001). Ces termites déprédateurs consomment une proportion importante de débris végétaux et sont présents dans les cultures (Wood, 1976 ; Wood \& Sands, 1978). Leur possibilité de stocker des aliments sous forme de meules à champignons souterraines leur permet de subsister dans les périodes difficiles (lors de la saison sèche).

Les résidus de culture restés au sol, source de nourriture, stimulent la recolonisation des champs par les termites champignonnistes (Wood et al., 1980 ; Tondoh, 1992 ; Mora et al., 1996 ; Sileshi et al., 2005). Par ailleurs, Wood et al., (1977 et 1980) au Nigeria sur le maïs, Kumar \& Veeresh (1990) en Inde sur le blé, Wood et al., (1987) au Yémen sur le coton ont montré que les attaques des termites sont peu importantes la première année (1-2\%), mais qu'elles évoluent avec le temps sur les parcelles non traitées. Au niveau du maïs, le mode d'attaque des Microtermes, Ancistrotermes, Amitermes, Pseudacanthotermes, Trinervitermes est similaire à ceux observés par Wood et al., (1980), Cowie et Wood (1989) et Cissé (1991). Cette attaque peut évoluer de façon externe sur les tiges entraînant la formation de galeries externes dites galeries tunnels (Grassé, 1949) cité par Cissé (1991).

Il existe peu de données sur les termites ravageurs de la culture de riz en Afrique, où les dégâts en général semblent peu importants (Breniere, 1983). En revanche, des données existent en Amérique latine, où les genres Syntermes et Cornitermes sont de véritables ravageurs de riz au Brésil avec des dégâts localisés au niveau des racines et du feuillage (Sands, 1973 ; Carmélia et al., 2003). Syntermes molestus peut être considéré comme le principal termite déprédateur du riz, pouvant détruire totalement ou partiellement le système racinaire (Dario \& Villela_Filho, 1998). Les plants de riz attaquées sont rabougries et ne portent généralement pas de panicules (Jacquot \& Courtois, 1983). Les attaques au niveau de la tige de riz sont en général superficielles et peu graves (Lee et Wood, 1971). Les dégâts causés par les termites sur le riz seront d'autant plus importants que la plante attaquée souffrira simultanément de la sécheresse ou du défaut de fertilité du sol (Betbeder, 1989).

L'importance des attaques des termites sur le maïs comparativement au riz pourrait s'expliquer par le fait que le riz arrive à supporter les vents et le stress hydrique (Smit \& Van den Berg, 2003 ; Riekert \& Van den Berg, 2003). Les attaques des termites démarrent dès le stade de plantules si bien que la culture de riz qui arriverait à supporter les premières attaques ne présenterait aucune réduction de la densité des plants et pourrait arriver à maturité (Logan et al., 1990 ; Pearce et al., 1995). II faut aussi, signaler que les superficies de riz pluvial emblavées par les paysans sont faibles et les termites trouveraient leurs aliments préférés en abondance en dehors de celles-ci ; dans la végétation pérenne qui entoure ces champs (Nyeko \& Olubayo, 2005).

\section{Conclusion}

L'objectif de cette étude était d'évaluer le rôle de certains termites dans la destruction des champs de riz et de maïs. Les termites rencontrés en milieu naturel sont souvent présent dans les champs de riz. Concernant les champs de maïs, seuls les termites champignonnistes subsistent. Les modalités d'attaque des termites sont très variables, elles portent généralement sur les racines et se poursuivent sur les tiges. Les 
termites de petites tailles (Microtermes $s p$, Ancistrotermes $s p$ et Amitermes $s p$ ) construisent des galeries tandis que les plus grands (Pseudacanthotermes $s p$ ) font des placages de terre sur les cultures qu'ils utilisent lors des attaques.

Les dommages provoqués par les termites dans les champs paysans méritent d'être étudiés de manière exhaustive afin d'élaborer des méthodes de lutte durable. Une attention particulière doit être accordée à la compréhension des méthodes traditionnelles de contrôle des termites en agriculture afin de réduire les quantités d'insecticides utilisées.

\section{Références citées}

Akpesse A. A., Kouassi P., Yapi A., Lepage M., Tano Y., \& Tahiri A., 2001.Influence des traitements insecticides sur les populations de termites nuisibles aux cultures de riz et de maïs en milieu de savane (Lamto et Booro-Borotou, Côted'Ivoire). Agron. Afr., 13 : 45-94.

Akpesse A. A., Kouassi P., Yapi A., Lepage M., Tano Y., \& Tahiri A., 2004. Impact des traitements insecticides sur les populations de termites sur la production de riz et de maïs en savane sudsoudanienne (Booro-Borotou, Côte-d'lvoire) : cas du Chlorpyrifos-éthyl et du Fipronil. Bioterre, 4: 15-35.

Betbeder M., 1989. Insectes nuisibles aux cultures vivrières d'Afrique, de Madagascar et de Mascareignes. CIRAT, Paris, France. 119 pp.

Boga J. P., Kouassi Ph., Yapi A., Tahiri A., \& Tano Y., 2000. Modification des propriétés des sols par les termites et impact des sols de termitières sur la production de maïs et de riz en savane de Côte-d'Ivoire. Sci. Nat. Agron. 24: 54-65.

Bignell D., \& Eggleton P., 2000. Termites in ecosystems. In: Termites: Evolution, Sociality, Symbioses, Ecology. (Abe T, Bignell D.E. \& Higashi M. (eds). Kluwer Academic Publisher, Amsterdam, pp. 363-387.

Breniere J., 1983. Les ravageurs du riz en Afrique de l'Ouest. CIRAD-IRAT, Montpellier, France. $87 \mathrm{pp}$.

Carmélia S. M., Vasconcellos A. \& Bandeira G.A., 2003. Termites in sugar cane in Northern Brazil: ecological aspects and pest status. Neotrop. Entomol. 3: 1-9.
Carter S. E., \& Murwira H.K., 1995. Spatial variability in soil fertility management and crop reponse in Mutoko Communal Area Zimbabwe. Ambio. 24: 77-79.

Cissé M., 1991. Contribution à l'étude de l'entomofaune du maïs (Zea mays) et lutte contre les termites. Mémoire de DEA, Université d'Abidjan, Côte-d'Ivoire. 67 pp.

Constantino R., 2002. The pest termites of South America: Taxonomy, distribution and statuts. J. Appl. Entomol. 126: 355-365.

Cowie R. H., \& Wood T.G., 1989. Damage to crops, forestry and rangeland by fungus-growing termites (Termitidae: Macrotermitinae) in Ethiopia. Sociobiology, $15: 139-153$.

Dario G. J. A. \& Villela Filho R. da S., 1998. Imidacloprid- Novo insecticida para o controle do cupium (Syntermes molestus) na cultura do arroz (Oryza sativa L). In: XVII Congresso Brasieiro de Entomologia Resumos. pp. 206 -222.

Diomandé T., 1983. Le peuplement en fourmis terricoles de deux forêts ombrophiles de Côted'Ivoire. Ann. Univ. Abidjan, E. 16 : 39-56.

Eggleton P., Bignell D.E., Sands W.A., Waite B., Wood T.G. \& Lawton J.H., 1995. The species richness of termites (Isoptera) under differing levels of forest disturbance in the Mbalmayo Forest Reserve, Southern Cameroon. J. Trop. Ecol. 11: 85-98.

Fall S., Sarr M., Rouland C. \& Brauman A., 2000. Effets de l'âge de la jachère et de la saison sur la densité et la diversité des termites. In : La jachère en Afrique tropicale Floret $\mathrm{Ch}$, et Pontanier R. (eds). John Libbey Eurotxt, Paris, pp. 259-267.

Fowler H. G. \& Forti L.C., 1990. Statuts and prospects of termite problems and control in Brazil. Sociobiology. 17: 45-56.

Fritsch E. \& Planchon O., 1987. Bassin versant de Booro-Borotou. Carte des sols à 1/2500. ORSTOM, Adiopodoumé.

Han S. H., Tokro G.P., Tano Y. \& Lepage M., 1998. Dégâts des termites dans les jeunes plantations de palmiers à huile en Côte-d'Ivoire : évaluations et méthodes de lutte. Plantations, Recherches, Développement, 5 (2): 119-123.

Iroko F. A., 1996. Les dégâts des termites. In : L'homme et les termitières en Afrique. Edition 
Karthala. pp 157-169.

Jacquot M. \& Courtois B., 1983. Le riz pluvial. Le technicien d'agriculture tropicale. Editions Maisonneuve Larose et A.C.C.T. Paris. Pp 134.

Kambhampati S. \& Eggleton P., 2000. Taxonomy and phylogeny of termites. In: Termites: Evolution, Sociality, Symbioses, Ecology. (Abe T, Bignell D.E. \& Higashi M. (eds). Kluwer Academic Publishers, Amsterdam, pp. 1-23.

Kooyman C., \& Onck R. F. M., 1987. Distribution of termite (Isoptera) species in South-western Kenya in relation to land use and morphology of their galleries. Biol Fert Soils, 3: 69-73.

Kouassi K. P., 1999. Structure et dynamique des groupes trophiques de la macrofaune du sol d'écosystèmes naturels et transformés de Côte d'Ivoire. Thèse d'Etat ès Sciences, Université de Cocody, Abidjan, 201 pp.

Kumar C. T. A., \& Veeresh G. K., 1990. Assessment of losses to wheat by Microtermes obesi Holmgren and its management. Social Insects: 203-207.

Lavelle P., Bignell D., Lepage M., Wolters V., Roger P., Inesi P., Heal O.W. \& Dhillion S., 1997. Soil function in a changing world: The role of invertebrate ecosystems engineers. Eur. J. Soil Biol. 33: 159-193.

Lee K.E. \& Wood T.G., 1971.Termites and soils. Academic press, London. $251 \mathrm{p}$.

Legendre P. \& Anderson M. J., 1999. Distancebased redundancy analysis: testing multispecies responses in multi-factorial ecological experiments. Ecol. Monographs. 69 (1): 1-24.

Legendre P. \& Gallagher E., 2001. Ecologically meaningful transformations for ordination of species data. Oecologia. 129: 271-280.

Lepage M., 1974. Les termites d'une savane sahélienne (septentrionale, Sénégal), peuplements, populations, consommation, rôle dans l'écosystème. Thèse de Doctorat de l'Université de Dijon, 334 pp.

Levieux J., 1967. Données préliminaires sur le peuplement en fourmis terricoles dans la savane de Lamto (Côte-d'Ivoire). La terre et la vie. 21 : 278-296.

Logan M.W., Cowie R.H. \& Wood T.G., 1990. Termite (Isoptera) control in agriculture and forest by nonchemical methods a review. Bull. Entomol. Res. 80: 309-330.

Mill A.E., 1992. Termites as agricultural pests in Amazonia, Brazil. Outlook Agric.21: 41-46.

Mora P., Rouland C. \& Renoux J., 1996. Foraging, nesting and damage caused by Microtermes subhyalinus (Isoptera: Termitidae) in a sugarcane plantation in the Central African Republic. Bull. of Entomol. Res, 86: 387-395.

Nyeko P. \& Olubayo F.M., 2005. Participatory assessment of farmers experiences of termite problems in agroforestry in Tororo District, Uganda. AGREN. 143: 1-15.

Pearce M.J., Logan J.W.M. \& Tiben A., 1995. Termites (Isoptera) from the Darfur region of Sudan with comments on their pest statuts. J. Arid Environment. 30: 197-206.

Pham D.T.L., 1990. Etude de la variation pluviométrique des stations de Bouna, Ferkessédougou, Touba, Lamto. In: Productivité des savanes de côte d'Ivoire, bases scientifiques pour une meilleure gestion de leurs ressources. Projet PNUD/UNESCO, N IVC/87/007, Note technique, $24 \mathrm{pp}$.

Raymundo S.A., 1986. Traditional pest control practices in West Africa. IRRI Newsletter, 11: 24-27.

Rice W.R., 1989. Analyzing tables of statistical tests. Evolution 43:223-225.

Riekert H.F. \& Van den Berg J., 2003. Evaluation of maize cultivars and rotations crops for resistance to damage by fungus-growing termites. Suid-Afrikaanse Tydskrift Plant Grond, 20: $72-75$.

Sands W.A., 1973. Termites as tree and crop pests. Pans. 19: 167-177.

Sands W.A., 1977. The role of termites in tropical agriculture. Outlook on agriculture. 9. 136-143.

Sarr M., 1999. Etude écologique des peuplements de termites dans les jachères et dans les cultures en zone soudano-sahélienne, au Sénégal. Thèse de Doctorat de $3^{\text {ème }}$ cycle de Biologie Animale. Université Cheikh Anta Diop de Dakar. 117 pp.

Sarr M., Agbogba C. \& Russel-Smith A., 2001. Effects of soil faunal activity and woody shrub on water infiltration rates in a semi-arid fallow of Senegal. Appl. Soil. Ecol. 16: 283-290. 
Sekamatte M.B., Ogenda-Latigo M.W. \& RussellSmith A., 2001. The effect of maize stover used as mulch on termite damage to maize and activity of predatory ants. Afr. Crop. Sci. J.: 9 (2) 411-419.

Séré Y., 1990. La striure et les autres maladies du maïs, au Burkina Faso. In : Lutte intégrée contre les ennemis des cultures vivrières dans le sahel. Bamako, Mali, Institut du Sahel, pp. 276-284.

Sileshi G., Mafongoya P.L., Kwesiga F. \& Nkunika P., 2005. Termite damage to maize grown in agroforestry systems, traditional fallows and monoculture on nitrogen-limited soils in eastern Zambia. Agric. Forest. Entomol., 7: 61-69.

Smit E. \& Van den Berg J., 2003: Effect of tillage methods on termite damage to maize in South Africa. Insect Sci. and its Appl. 4: 153-156.

Sokal, R.R. \& Rohlf, F.J., 1995, Biometry: the principles and practice of statistics in biological research, Edition Freeman and Company, pp. 887.

Tano Y., 1993. Les termitières épigées d'un bassin versant en savane soudanienne: répartition et dynamique des nids, rôle sur les sols et sur la végétation. Thèse d'Etat ès Sciences, Université Nationale de Côte-d'Ivoire, Abidjan, 250 pp.

Tondoh E.J., 1992. Influence de la mise en culture sur le peuplement de la macrofaune du sol en moyenne Côte-d'lvoire. Mémoire de DEA, Université Abidjan. Côte-d'Ivoire. 66 pp.

Umeh V.C., Waliyar F., Traoré S., Chaibou I.M., Omar B. \& Detognon J., 2001. Farmer's

opinions and influence of cultural practrices on soil pest damage to groundnuts in West Africa. Insect Sci. and its Appl. 3: 257-265.
Wardell D.A., 1987. Control of termites in nurseries and young plantations in Africa: established practices and alternative courses of action. Commonwealth For. Revue, 66: 77-89.

Wood T. G. 1976. The role of termites (Isoptera) in decomposition processes. In: the role of terrestrial and aquatic organisms in decomposition processes. Eds J.M. Anderson \& Macfadyen. Blackwell, Oxford. England. pp. 145-168.

Wood T. G. 1996. The agricultural importance of termites in the tropics. Agr. Zool. Rev. 7: 117-155.

Wood T.G. \& Sands W.A., 1978. The role of termites in ecosystems. In: Production Ecology of Ants and Termites (M.V. Brian, Ed), London, England. pp. 245-292.

Wood T.G. \& Pearce M.J., 1991. Termites in Africa: the environmental impact of control measures and damage to crops, trees, rangeland and rural buildings. Sociobiology., 19: 221-234.

Wood T.G., Johnson R.A. \& Ohiagu C.E., 1977. Populations of termites (Isoptera) in natural and agricultural ecosystems in Southern Guinean savanna near Mokwa, Nigeria. Geotrop. Econ. Trop., 1: 139-148.

Wood T.G., Johnson R.A., \& Ohiagu C. E., 1980. Termite damage and estimation of damage loss in yield and termite (Microtermes) abundance at Mokwa. Trop. Pest Manag., 26: 241-253.

Wood T.G., Bednarzik M., \& Aden H., 1987. Damage to crop by Microtermes najdensis (Isopteratermitinae) in irrigated semi-desert areas of the red sea coast. 1- The Tihama region of the Yemen Arab Republic. Trop. Soil. Manag., 33: 142-150. 